\title{
An Intelligent Approach to Reducing Tobacco Smoking among Adolescents: Health Risk Expert Approach
}

\author{
Hyellamada Simon \\ Department of Computer \\ Science, Federal Polytechnic, \\ Mubi, Nigeria.
}

\author{
Benson Yusuf Baha \\ Department of Computer \\ Science, Taraba State \\ University, Jalingo, Nigeria.
}

\author{
Nachandiya Nathan \\ Department of Computer \\ Science, Adamawa State \\ University, Mubi, Nigeria.
}

\begin{abstract}
Tobacco smoking causes health challenges both nationally and internationally, and there is need to reduce tobacco consumption. To achieve this, the researchers used Case Based Reasoning (CBR) methodology and Nearest Neighbour (NN) similarity technique in developing a web-based expert system to assist in reducing the rate of tobacco consumption among adolescents. In the cause of the research, 20 related diseases to tobacco smoking were identified and stored in the knowledge based; these data were used to test 3 new cases and their inherent individual level associated to it. The new cases are tuberculosis, laryngeal cancer (throat cancer), and asthma with highest risk values of 1.0, 0.8, and 0.9 respectively. The results provided a procedure to assist adolescents (smokers) minimize tobacco consumption. The system also offers some home remedies to help them deal with nicotine withdrawal syndrome. Therefore, adolescents are advised to quit smoking in order to avoid serious health problems in the future.
\end{abstract}

\section{General Terms}

Artificial Intelligence, Case-Based Reasoning, Nearest Neighbor, and Expert System

\section{Keywords}

Adolescent, Artificial Intelligence, Case-Based Reasoning, Nearest Neighbor, Cigarette, Expert System, Nicotine, and Tobacco

\section{INTRODUCTION}

Tobacco smoking is a serious issue nationally and internationally which affects the health of users and causes public pollution. Despite warnings as written on the cigarette pack that "tobacco smoking is dangerous to health", the number of smokers seem to be increasing. According to World Health Organization-WHO (2015), tobacco remains the only legal drug which kills most of its users when used exactly as recommended by the manufacturers. They also estimated that tobacco use (smokeless and smoke) is presently responsible for the death of almost $6,000,000$ people worldwide every year and most of them happened prematurely. The effects of tobacco use are seriously harmful to virtually every organ of the human body (Kholer, Ward, \& McCarthy, 2011).

Mohasoa (2010) is of the view that most adolescents start using drugs as early as 12 years of age. This wild behavior of tobacco use among adolescents usually starts with smoking cigarettes in school toilets during school break times, and then proceeds to use other substance such as marijuana, alcohol, cannabis and hard drugs. Adolescents use tobacco for a number of reasons and the contributing factors include their growing stage, peer group pressure, stress relief and family problems. They seem not to consider the long-term effect of this tobacco on their lives. Many of these young smokers may die eventually from a smoking related disease. Examples of negative health consequences for youth who smoke includes: Cough increased rate and severity of respiratory illnesses; Smoking harms young people's physical fitness in terms of endurance and performance; the younger a person starts smoking, the more likely to become strongly addicted to nicotine. Smoking may cause mental health problems, such as depression among adolescents which will affect their academic performance and end up as school drop-out, thereby adding to the rising rate of unemployment in the society (Mohasoa, 2010; Kabulwa, 2011).

Though efforts were made by many researchers to help adolescents quit smoking, but the number of youth involved in smoking kept on increasing. Hence we proposed an intelligent approach to assist in reducing tobacco consumption among adolescents. The researchers used Case Based Reasoning (CBR) methodology and Nearest Neighbour (NN) similarity technique to design an intelligent system for predicting risks involved in tobacco smoking and related diseases prone to adolescents involved in such acts. This will eventually lead to reduction or possibly total quit of tobacco smoking.

Expert System (ES) and Intelligent Decision Support System (IDSS) help a lot in medical fields. An ES, also called a Knowledge Base System (KBS) is a computer program that contains most of the knowledge of a specific subject of one or more human experts (Hole and Gulhane, 2014).

The proposed system is a web based Expert System (ES), which would assist the adolescents smokers in predicting individual risks level of association with tobacco related disease based on their current symptoms. Hence, provides procedure for quitting smoking, and some home remedies to assist them deal with nicotine withdrawal syndrome.

\section{LITERATURE REVIEW}

Prochaska et al. (2000) presented an interactive Expert System intervention for a general public smoking cessation. The Expert System was enhanced with telephone calls for counselling and feedback. Mary et al. (2008) also developed a prototype Clinical Decision Support System (CDSS) for smoking cessation which was used for tobacco smoking counselling by clinicians. Also Brunette et al. (2015) developed a web-based motivational decision-support system designed to help disadvantaged smokers into an effective tobacco cessation treatment.

Mathew, Shilu, Hamed and Qadri (2015) used a hybrid approach of Rule-based Reasoning (RBR) and Case-based Reasoning (CBR) to develop Intelligent Clinical Support System for medical diagnosis of disorders of the nervous system (neurological disorder) as a web-based expert system. The diagnosis made by the system was evaluated to be correct 
as those made by the human expert; this improves the effectiveness of diagnosis. Haris (2012) developed an Intelligent Medical Decision Support System using Fuzzy Logic and CBR methodology for diagnosis of patient's condition based on current symptoms of hepatitis. The system was validated and evaluated which shows that it was reliable and can be used to assist doctors throughout the diagnosis process.

Salem, Roushdy and HodHod (2005) used CBR methodology to develop a case-based expert system model that support diagnosis of heart diseases. The structure of knowledge was represented over cases. The techniques for retrieval process used by their system include; nearest-neighbor and induction techniques. The system performance was evaluated by a cardiologist by practically testing it for 13 new cases and the system successfully estimated their correct diagnosis.

Patel and Virparia (2013) presented an expert system using rule-based reasoning for diagnosis and treatment of viral infections which was deployed on a web server. The diagnosis provided by this system was proven accurate and faster due to many professionals knowledge used in developing the knowledge base of the system.

\section{OVERVIEW OF CBR PROCESS}

De-mantaras et al. (2005) defined CBR as a field of AI which is inspired by human reasoning. That is, the approach emphasized the role of previous experience to solve future problem. An experience (a case) generally contains a problem, a diagnosis/classification, a solution and its results. New problems are solved by reusing and if necessary adapting the solutions to similar problems that were solved in the past. According to Ekerholt et al. (2014), CBR is established on the assumption that situations mostly repeats with regularity, this means what was done in a previous situation is most likely to be appropriate in a similar situation. This is the same kind of problem solving by humans. Schank (1983) revealed that foundation of CBR was established from the Dynamic Memory model, which states that human understanding, remembering, experiencing and learning are combined processes altogether which cannot be separated. It means that humans understand by integrating new experiences with what they know already, which forms theoretical foundations of the CBR process. Salem et al. (2005) stated four main steps in the developing any CBR systems. These include:

i) Case representation and identifying key features,

ii) Indexing and retrieving similar cases from the case based,

iii) Measuring case similarity to select the best match, and

iv) Modifying the existing solution to fit the new problem.

\subsection{Application of CBR in Medical Domain}

According to Ekerholt et al. (2014), cases are generally used in day-to-day clinical practices where professional records are required to be kept for each patient; such as symptoms description, treatments and result of those treatments. This means there is a large collection of recorded medical cases that a Case-Based reasoner could use in building extensive case bases that can be used to learn from. The process of reasoning of a CBR system is similar to the reasoning of physicians in the process of diagnosing a patient. In order to diagnose a new patient a physician mostly goes back to old cases to look for similarities and possible indications as to what is wrong with the patient. If a similar case is found, the physician can use the conclusion of that case as a recommendation for the new situation. Since medical field is extremely complex, and there is still a lot to be known about the human body, a medical Case-Based reasoner is required to be capable of handling difficult cases with possible contradicting symptoms that could be as a result of multiple fundamental causes. A Case-Based reasoner can also justify their conclusions, by referring to past cases where the same solution was successful. CBR has shown to be successful in a number of different medical applications (Begum, 2009).

\subsection{System Architecture}

The system architecture clearly describes how different components of the system can interact with each other, and with the CBR technique. Aamodt and Plaza (1994) designed a CBR structure which was reused to develop the architecture of this system as shown in Figure 1 below.

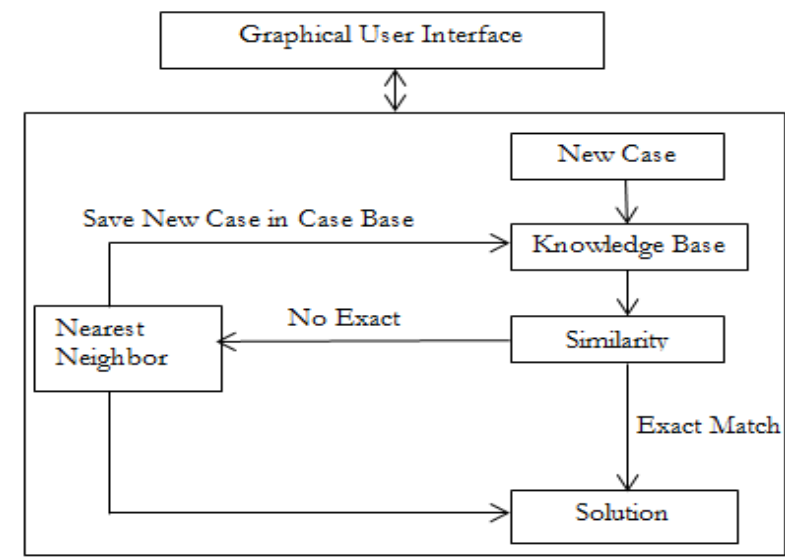

Figure 1: The System Architecture

Figure 1 above shows how inputs are supplied to the CBR system as symptoms and the system performs similarity matching operations between the new case and the cases saved in the knowledge based, when it gets exact match the result is displayed for reuse while if there is no exact match, $\mathrm{NN}$ technique is applied to search for similar results that can be adjusted to suit the new case. Finally, the new case is then retained in the knowledge based for future reuse.

\subsection{Risk Prediction and Case Retrieval Process}

Relevant information (symptoms) would be collected from the patient in the form of indexed features (see table 1 below). These features are compared with the features of the cases saved in the knowledge based; this is called case retrieving process, where cases similar to the new case are retrieved. When the system retrieved these cases after a successful matched, the next step is to reuse the case with exact match, otherwise $\mathrm{NN}$ technique is used to perform both local and global similarity evaluation in order to retrieve list of similar cases to the new case, then reuse the case with highest similarity value as a suggested solution to the new case.

The Local similarity matching computes the similarity between each feature of the new case and existing cases using equation 1 below.

$$
\operatorname{sim}\left(f_{i}^{N}, f_{i}^{R}\right)=\frac{\sum_{i=1}^{n}\left(f_{i}^{N} * f_{i}^{R}\right)}{\sum_{i=1}^{n} \sqrt{f_{i 2}^{N}} * \sum_{i=1}^{n} \sqrt{f_{i 2}^{R}}}
$$


The evaluation of the global similarity is done using equation 2 below, based on the local similarity values computed earlier and the attribute weights.

$$
\begin{aligned}
\operatorname{Similarity}\left(\operatorname{Case}^{N}, \operatorname{Case}^{R}\right) & \\
& =\frac{\sum_{i=1}^{n} w_{i} * \operatorname{sim}\left(f_{i}^{N}, f_{i}^{R}\right)}{\sum_{i=1}^{n} w_{i}}
\end{aligned}
$$

Where: $N=$ new case, $R=$ retrieved case, $f_{i}^{N}=$ values of features of new case, $f_{i}^{R}=$ values of features of retrieved cases, $w_{i}=$ essential weight attached to each feature, $i=$ individual feature from 1 to $\mathrm{n}$, and $i_{2}=$ total values of all features of a case (Kolodner, 1993; Salem et al., 2005; Ahmed et al., 2008; Begum, 2009).

The indexed features in table 1 below are used to assign weights by the expert according to the importance of individual feature to each disease. Though, the importance of each feature depends on the uniqueness of the feature in each disease. When a feature is common among all diseases it will then have the lowest weight, while if a feature is very unique in a disease and do not appear in any other disease, it will have the highest weight. These weights are assigned by the professional for accuracy of results.A similarity value 1 is an indication that a feature in a target case matched a feature in old cases, and similarity value 0 is an indication that a feature has no match.

\subsubsection{Algorithm of the System}

Step 1: Input all feature weights.

Step 2: Use equation 1 above to compute local similarity of a new case.

Step 3: Use equation 2 above to compute global similarity of a new case based on the local similarity computed earlier.

Step 4: The most similar cases are retrieved after computation of global similarity; a case with highest similarity value is reuse as a suggested solution to the current problem.

Step 5: The new solution is then stored in the knowledge based for future reuse.

\subsection{Data Modeling}

This paper identified 20 diseases associated with tobacco smoking as: Angina pectoris, Asthma, Atherosclerosis, Acute myelogenous leukemia, Barrette's esophagus, Bladder cancer, Bronchitis, Cervical cancer, chronic obstructive pulmonary disease, Emphysema, Gastro-esophageal reflux disease, Kidney cancer, Laryngeal cancer (throat cancer), Otitis Media, Peptic ulcer, Periodontal diseases, Pneumonia, Rhinitis, Sinusitis, and Tuberculosis which were used for the system development and stored in the knowledge based of the system. The data were gotten from related literatures (Oxford Medical Dictionary, 2002; U.S. Department of Health and Human Services, 2010; Action on Smoking and Health-ASH, 2012; ASH, 2013; Schmidt, 2014).

The patient supplies input data to the system in the form of symptoms they experienced. The weighted indexed feature generally contains the symptoms which are used in solving the new case by performing similarity matching. Though some patients may or not show all the symptoms for a particular disease at the same time. The weighted indexed features are shown in the table 1 below.

\begin{tabular}{|c|c|c|c|}
\hline Weight & Features & $\begin{array}{l}\text { Data } \\
\text { type }\end{array}$ & Value \\
\hline 7 & Bad breath & Boolean & Yes/No \\
\hline 9 & Changes in voice & Boolean & Yes/No \\
\hline 7 & Chest congestion & Boolean & Yes/No \\
\hline 2 & Chest pain & Boolean & Yes/No \\
\hline 7 & Cough & Boolean & Yes/No \\
\hline 9 & Coughing up blood & Boolean & Yes/No \\
\hline 7 & Decreased sense of smell & Boolean & Yes/No \\
\hline 6 & Difficulty breathing & Boolean & Yes/No \\
\hline 6 & Difficulty swallowing & Boolean & Yes/No \\
\hline 6 & Ear pain & Boolean & Yes/No \\
\hline 2 & Fatigue & Boolean & Yes/No \\
\hline 4 & Fever & Boolean & Yes/No \\
\hline 9 & Loss of appetite & Boolean & Yes/No \\
\hline 9 & Night sweat & Boolean & Yes/No \\
\hline 5 & Persistent cough & Boolean & Yes/No \\
\hline 3 & Shortness of breath & Boolean & Yes/No \\
\hline 9 & Sore throat that doesn't heal & Boolean & Yes/No \\
\hline 5 & Weight loss & Boolean & Yes/No \\
\hline 7 & Wheezing & Boolean & Yes/No \\
\hline
\end{tabular}

Table 1: Table of the weighted indexed feature

\section{RESULTS}

List of symptoms (features) are provided for the user to select from. After selecting all the required features, the system immediately retrieved list of similar matched cases with their similarity values for reuse. The test results of the system and the graphical representation of the results are displayed in figures 2 to 7 below. However, table 2 below revealed the procedure to assist adolescents to quit smoking and some home remedies to deal with nicotine withdrawal syndrome as part of the results.

Table 2: Procedure for quitting smoking and home remedies to deal nicotine withdrawal syndrome

\begin{tabular}{l}
\hline Procedure for quitting smoking \\
\hline 1. It is extremely important for a smoker to make up his/her \\
mind to quit smoking and set a quit date immediately. \\
2. Such person should stay away from smokers and places \\
where people smoke. \\
3. Keep company of people that will encourage and support \\
their decision to quit smoking. \\
4. Throw away all cigarettes and lighter, then wash all cloths \\
and clean apartment from all tobacco smell. \\
5. If it is hard to get used to not holding something or \\
\hline
\end{tabular}


not having a cigarette in mouth, then use sugar-free gum, mints, toothpicks, or lollipops.

6. Such person should keep themselves busy by listening to their favourite music, or by exercising their body.

7. If their body is addicted to nicotine, such person may experience some withdrawal syndrome when quitting smoking, these includes: headaches or stomach-aches, crabbiness, anxiety, or depression, lack of energy, dry mouth or sore throat, etc.

Note: These symptoms will go away after sometimes, so be patient not to smoke again. Instead use the home remedy below to fight nicotine withdrawal, or talk to a Doctor about other treatment options, like use of nicotine replacement; gums, patches, inhalers or nasal spray.

Home remedies to deal with nicotine withdrawal syndrome

1. Water is a natural detoxifier. Therefore, always drink enough water to help in fighting addictions.

2. As one tries to quit smoking, such person may start to feel nauseous. To fight that nausea, one can take ginger, either in tablets, capsules or as a tea. It will calm stomach and helps to overcome the nausea.

3. As a person fights the toxins in their body, take multivitamins, particularly those comprising Vitamins A, $\mathrm{C}$, and $\mathrm{E}$ can help in repairing the body and giving it the necessary nutrients needed to expel toxins.

4. If a person wants to specifically fight cravings to smoke, then add a spoonful of ginseng powder to their juice, porridge or soup in the morning. It can help fight their cravings all day.

5. Drink grape juice daily. The acids in the juice are natural detoxifiers which will revitalize the system and cleanse out the toxins faster.

\subsection{Test Result 1}

\begin{tabular}{|c|c|c|}
\hline$s / n$ & Disease & Similarity Values \\
\hline 1 & Tuberculosis & 1 \\
\hline 2 & Bronchitis & 0.52 \\
\hline 3 & Preumonia. & 0.52 \\
\hline 4 & Kidney cancer & 0.37931034482759 \\
\hline 5 & Sinusitis. & 0.2 \\
\hline 5 & Asthma. & 0.32142857142857 \\
\hline 7 & Acute myelogenous leukemia & 0.095238095236095 \\
\hline 8 & Laryngeal cancer (throat cancer) & 0.11904761904762 \\
\hline 9 & Chronic obstructive pulmonary disease & 0.076923076923077 \\
\hline 10 & Peptic ulcer. & 0.076923076923077 \\
\hline 11 & Angina Pectoris & 0.1 \\
\hline 12 & Barrette's Esophagus & 0.11764705882353 \\
\hline 13 & Gastro-esophageal Reflux Disease. & 0.045454545454545 \\
\hline 14 & Atherosclerosis. & 0.0416666666666667 \\
\hline
\end{tabular}

Figure 2: Test result for prediction of Tuberculosis

From figure 2 above, the test result shows that the patient is at risk of associating with Tuberculosis with the highest similarity value of 1.0 as represented in the graph below:

\section{Similarity values}

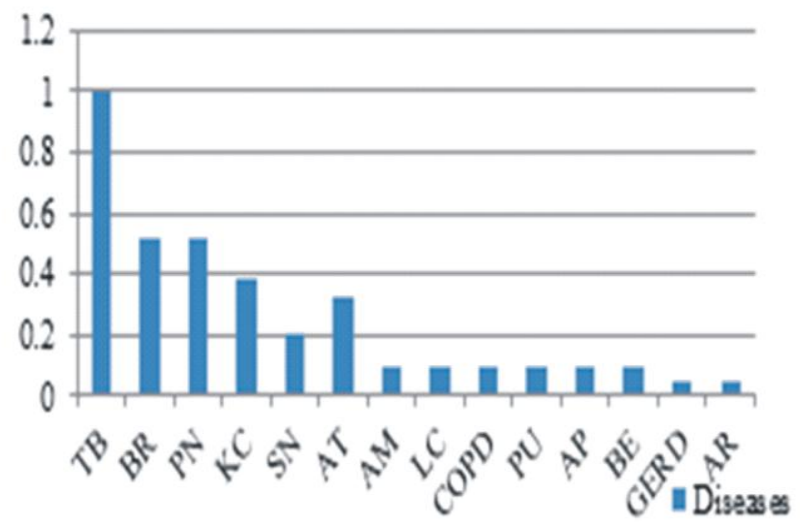

Key: TB: Tuberculosis, BR: Bronchitis, PN: Pneumonia, KC: Kidney cancer, SN: Sinusitis, AT: Asthma, AM: Acute myelogenous leukemia, LC: Laryngeal cancer, COPD: Chronic obstructive pulmonary disease, PU: Peptic ulcer, AP: Angina pectoris, BE: Barrette's esophagus, GE.2RD: Gastro-esophageal reflux disease, AR: Atherosclerosis.

Figure 3: Graphical representation for prediction of Tuberculosis

From figure 3 above, the graphical representation clearly shows that the patient is at risk of associating with Tuberculosis with the highest similarity value of 1.0. Therefore, the patient is advised to quit smoking using the procedure in table 2 above.

\subsection{Test Result 2}

\begin{tabular}{|c|c|c|}
\hline $5 / 11$ & Disease & Similarity Values \\
\hline 1 & aryngeal cancer (throat cancer) & 0.78571428571429 \\
\hline 2 & Gastro -esophageal Refux Disease. & 0.3409090909090909 \\
\hline 3 & khinits. & 0.090909090909091 \\
\hline 4 & Barrette's Esophagus & 0.352244117647059 \\
\hline 5 & Dotts Media, & 0.25 \\
\hline 6 & Sinusits. & D.1090909090909091 \\
\hline 7 & Kidney cancer & 0.17241379310345 \\
\hline$\beta$ & Tuberculosis & 0.111111111111111 \\
\hline 9 & Pepticulcer, & 0,0769230769923077 \\
\hline 10 & Chronic obstuctive pummonary disease & 0.0769230769923077 \\
\hline
\end{tabular}

Figure 4: Test result for prediction of Laryngeal cancer (throat cancer)

From figure 4 above, the test result shows that the patient is at risk of associating with Laryngeal cancer (throat cancer) with the highest similarity value of 0.8 as represented in the graph below: 
Similarity values

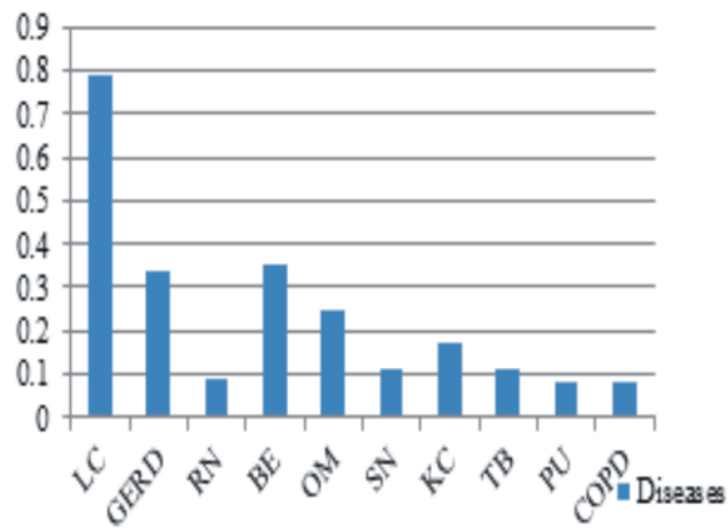

Key: LC: Laryngeal cancer, GERD: Gastro-esophageal reflux disease, RN: Rhinitis, BE: Barrette's oesophagus, OM: Otitis media, SN: Sinusitis, KC: Kidney cancer, TB: Tuberculosis, $P U$ : Peptic ulcer, COPD: Chronic obstructive pulmonary disease.

Figure 5: Graphical representation for prediction of Laryngeal cancer (throat cancer)

From figure 5 above, the graphical representation clearly shows that the patient is at risk of associating with Laryngeal cancer (throat cancer) with the highest similarity value of 0.8 . Therefore, the patient is advised to quit smoking using the procedure in table 2 above.

\subsection{Test Result 3}

\begin{tabular}{|l|l|l}
\hline S/II & Disease & Similarity Values \\
\hline 1 & Asthma, & 0.92857142857143 \\
\hline 2 & Chronic obstructive pulmonary disease & 0.26153846153846 \\
\hline 3 & Bronchits & 0.48 \\
\hline 4 & Pneumonia. & 0.36 \\
\hline 5 & Tuberculosis & 0.2 \\
\hline 5 & Angina Pectoris & 0.175 \\
\hline 7 & Sinustis. & 0.12727272727273 \\
\hline 3 & Atherosclerosis. & 0.10416666666667 \\
\hline 3 & Acute myelogenous leukemia & 0.079365079365079 \\
\hline 10 & Emphysema & 0.15789473684211 \\
\hline 11 & Barrette's Esophagus & 0.11764705882353 \\
\hline 12 & Kidney cancer & 0.065965517241379 \\
\hline 13 & Gastro-esophageal Reflux Disease. & 0.045454545454545 \\
\hline
\end{tabular}

Figure 6: Test result for prediction of Asthma

From figure 6 above, the test result shows that the patient is at risk of associating with Asthma with the highest similarity value of 0.9 as represented in the graph below:

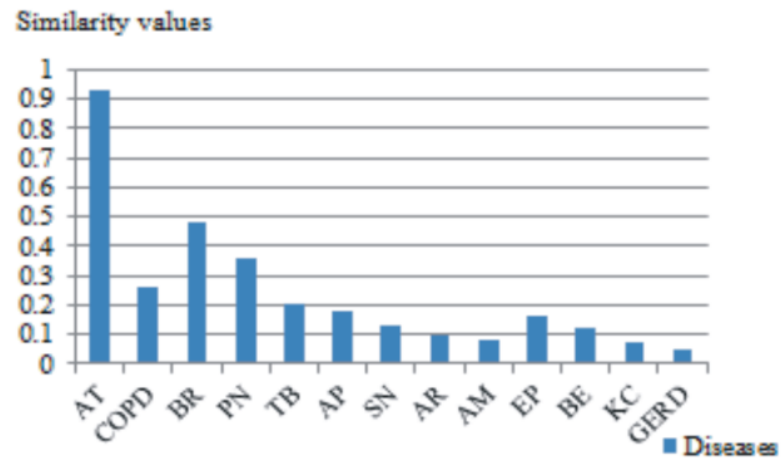

Key: AT: Asthma, COPD: Chronic obstructive pulmonary disease, BR: Bronchitis, PN: Pneumonia, TB: Tuberculosis, AP: Angina pectoris, SN: Sinusitis, AR: Atherosclerosis, AM: Acute myelogenousleukemia, EP: Emphysema, BE: Barrette's oesophagus, KC: Kidney cancer, GERD: Gastro-esophageal reflux disease.

Figure 7: Graphical representation for prediction of Asthma

From figure 7 above, the graphical representation clearly shows that the patient is at risk of associating with Asthma with the highest similarity value of 0.9 . Therefore, the patient is advised to quit smoking using the procedure in table 2 above.

\section{DISCUSSION}

The intelligent system was tested against 3 cases which were selected at random as shown in figures 2 to 7 above. From the above test results, figures 2 and 3 showed result of patient with symptoms, such as: chest pain $=2$, coughing up blood $=$ 9 , fatigue $=2$, fever $=4$, loss of appetite $=9$, night sweat $=9$, persistent cough $=5$, and weight loss $=5$. The system matched these features values with the cases stored in the knowledge base, and then displayed the list of all similar cases with their similarity values, thereby placing the most similar case with highest value as first in the list. Therefore, the result showed that the patient is at high risk of associating with Tuberculosis with the highest similarity value of 1.0, and is advised to quit smoking using the procedure provided in table 2. Finally, the result of the diagnosis is saved in the knowledge based of the system for future reuse.

Figures 4 and 5 above, showed result of patient with symptoms, such as: changes in voice $=9$, cough $=7$, difficulty swallowing $=6$, ear pain $=6$, sore throat that doesn't heal $=9$, and weight loss $=5$. The system matched these features values with the cases stored in the knowledge base, and then displayed the list of all similar cases with their similarity values, thereby placing the most similar case with highest value as first in the list. Therefore, the result showed that the patient is at high risk of associating with Laryngeal cancer (throat cancer) with the highest similarity value of 0.8 , and is advised to quit smoking using the procedure provided in table 2. Finally, the result of the diagnosis is saved in the knowledge based of the system for future reuse.

Figures 6 and 7 above, showed result of patient with symptoms, such as: chest congestion $=7$, chest pain $=2$, fatigue $=2$, persistent cough $=5$, shortness of breath $=3$, wheezing $=7$. The system matched these features values with the cases stored in the knowledge base, and then displayed the list of all similar cases with their similarity values, thereby placing the most similar case with highest value as first in the list. Therefore, the result showed that the patient is at high risk 
of associating with Asthma with the highest similarity value of 0.9 , and is advised to quit smoking using the procedure provided in table 2. Finally, the result of the diagnosis is saved in the knowledge based of the system for future reuse.

\section{CONCLUSION}

Intelligent System was developed as an attempt to provide assistance to adolescent smokers, where CBR method has proven to be an effective tool for prediction of smoker's individual level of health risks associated to tobacco smoking based on current symptoms. In this research, 20 diseases associated to tobacco smoking were identified and stored in the knowledge based of the system which were used to test for 3 new cases; the system accurately predicted their risks of associating with the following tobacco related diseases: tuberculosis, laryngeal cancer (throat cancer), and asthma with the highest risk values of $1.0,0.8$, and 0.9 respectively. The results also provided a procedure (see table 2 above) to assist smokers quits smoking, and some home remedies to help deal with nicotine withdrawal syndrome. Therefore, smokers are advised to use the findings of this research to help them quits smoking in order to avoid serious health challenges in future. This will in turn gradually reduce the number of adolescent smokers.

This research work can be improved further by integrating Case Based Reasoning and Artificial Neural Network methodologies to develop hybrid intelligent system for effective prediction and treatment of nicotine addiction among past and current smokers.

\section{REFERENCES}

[1] Aamodt, A., \& Plaza, E. 1994. Case-based reasoning: Foundational issues, methodological variations, and System approaches. AI Communications. 7. 39-59.

[2] Action on Smoking and Health. 2012. ASH Research Report on Tobacco and Oral Health. Retrieved 19th January, 2016, from http://www.ash.org.uk/files/documents/ASH_598.pdf

[3] Action on Smoking and Health. 2013. ASH Research Report on Smoking and Cancer. Retrieved 19th January, 2016, http://www.ash.org.uk/files/documents/ASH_109.pdf

[4] Ahmed, M. U., Begum, S., Funk, P., Xiong, N., \&Scheele, B. V. 2008. Case-Based Reasoning for diagnosisof stress using enhanced cosine and fuzzy similarity.Journal of IBaI Publishing. 1(1). 3-19.

[5] Begum, S. 2009. A case-based reasoning system for theDiagnosis of individual sensitivity to Stress in psychophysiology. Malardalen University Press Licentiate Theses. ISBN 978-91-86135-25-6.

[6] Brunette, M. F., Gunn, W., Alvarez, H., Finn, P. C.,Geiger, P., Ferron, J. C., McHugo, G.J. 2015. A prepost pilot study of a brief, web-based intervention toengage disadvantaged smokers into cessation treatmentAddiction Science \& Clinical Practice. 10(3).Doi:10.1186/s13722-015-0026-5.

[7] De-mantaras, R. L., Mcsherry, D., Bridge, D., Leake, D.,Smyth, B., Craw, S., ..., Watson, I. 2005. The Knowledge Engineering Review. Cambridge University Press.00(0).1-2. Doi:10.1017/S000000000000000.

[8] Ekerholt, M., Folleso, S. L., Heimark, O. 2014. A Systemfor Conversational Case-Based Reasoning in Multiple Disease Medical Diagnosis. Master's Thesis, Norwegian University of Science and Technology.
[9] Haris, M. F. 2012. Diagnosing hepatitis using hybridfuzzy-CBR. Master degree Dissertation. University Utara Malaysia.

[10] Hole, K. R., \& Gulhane, V. S. 2014. Rule-Based ExpertSystem for the Diagnosis of Memory Loss Diseases. IJISET. 1(3). 2348-7968.

[11] Kabulwa, M. N. 2011. Prevalence, perceived factors andknowledge on effects of tobacco use on oral health among secondary school students in dares salaam. Master's degree Dissertation. Muhimbili University of Health and Allied Sciences.

[12] Kohler, B. A., Ward, E., McCarthy, B. J. 2011. Annualreport to the nation on the status of cancer, 19752007, featuring tumors of the brain and other nervous system. JNatl Cancer Inst. 103(9). 714-736.

[13] Kolodner, J.L. 1993. Instructional design: Case Base Reasoning. Morgan Kaufmann Publishers, San Mateo, CA.

[14] Mary, T. W., Kaplan, B., Connoly, S. W., Michel, G.,Shiffman, R. N., \& Flynn, B. S. 2008. Developing a Decision Support System for Tobacco Use Counseling Using Primary Care Physicians. Inform Prim Care. 16(2).101-109.

[15] Mathew, S. Shilu, M., Hamed, M. H., \& Qadri, I. 2015. A web based decision support system driven for the Neurological disorders. IJERGS. 3(4).

[16] Mohasoa, I. P. 2010.Substance abuse among male adolescents. Master's degree Dissertation. University of South Africa.

[17] Oxford Medical Dictionary. 2002. Oxford University press Britain. (6th ed.)

[18] Patel, M. \& Virparia, P. 2013. Rule Based Expert System for Viral Infection. IJARCSSE. 3(5).

[19] Prochaska, J., O., Velicer, W. F., Fava, J. L., Ruggiero,L., Laforge, R. G., Rossi, J. S., Lee, P. A. 2001. Counselor and Stimulus Control Enhancements of a Stage-Matched Expert System Intervention for Smokers in a Managed Care Setting. Cancer Prevention Research Center. University of Rhode Island. 32. 23-32. doi:10.1006/pmed.2000.0767.

[20] Salem, A. M., Roushdy, M., \&HodHod, R. A. 2005. A case based expert system for supporting diagnosis of heart diseases. AIML Journal. 5(1).

[21] Schank, R. C. 1983. Dynamic memory: A theory of reminding and learning in computers and people. Cambridge University Press.

[22] Schmidt, L. 2014. Health harms from smoking and other tobacco use. Campaign for Tobacco-Free Kids. Retrieved from http://www.tobaccofreekids.org/facts_issues/fact sheets/toll/products/.

[23] U.S Department of Health and Human Services 2010.The Health Effect of Tobacco. Retrieved $19^{\text {th }}$ January, 2016, from www.cdc.gov/tobacco/data_statistics/sgr/2010/consumer _booklet/pdfs/consumer.pdf

[24] World Health Organization 2015.Global Report on Trends in Prevalence of Tobacco Smoking. WHO Library Cataloguing-in-Publication. Publication Number 9789241564922 (NLM classification: WM 290). 